\title{
A REABILITAÇÃO DE PESSOAS COM DEFICIÊNCIA ATRAVÉS DO DESPORTO ADAPTADO
}

\author{
MS. VINÍCIUS DENARDIN CARDOSO \\ Mestre em Actividade Física Adaptada - Faculdade de Desporto da \\ Universidade do Porto, Portugal \\ Graduado em Educação Física - Universidade Federal de Santa Maria, \\ (Santa Maria - Rio Grande do Sul - Brasil) \\ e-mail: vinicardoso@yahoo.com.br
}

\begin{abstract}
RESUMO
O desporto é um importante meio para a reabilitação física, psicológica e social de pessoas com algum tipo de deficiência. Trata-se de modificações e adaptações em metodologias, regras, materiais para a participação de pessoas com deficiências. O objectivo deste estudo foi realizar uma revisão de literatura sobre a reabilitação de pessoas com deficiência através do desporto adaptado, baseada em referências nacionais e internacionais obtidas através da base de dados da CAPES, Pubmed, Scielo e na base de dados da biblioteca da Faculdade de Desporto da Universidade do Porto - Portugal. Observou-se que os benefícios da prática desportiva são evidenciados por grande parte de estudiosos da área do desporto adaptado e contribuem para a qualidade de vida desta população.
\end{abstract}

PALAVRAS-CHAVE: Reabilitação; desporto adaptado; prática desportiva; pessoas com deficiência. 


\section{INTRODUÇÃO}

Cada vez mais observa-se a prática de actividades desportivas por pessoas com deficiência, a busca da melhoria da qualidade de vida nos últimos anos fez com que uma grande quantidade de pessoas com deficiência buscassem esta prática, visando estimular suas potencialidades e possibilidades, em prol de seu bem-estar físico e psicológico.

O desporto adaptado surgiu como um importante meio na reabilitação física, psicológica e social para pessoas com algum tipo de deficiência, consiste em adaptações e modificações em regras, materiais, locais para as atividades possibilitando a participação das pessoas com deficiências nas diversas modalidades esportivas (DUARTE; WERNER, 1995), e também pode ser definido como esporte modificado ou especialmente criado para ir ao encontro das necessidades únicas de indivíduos com algum tipo de deficiência (GORGATTI; GORGATTI, 2005).

A oportunidade da prática desportiva para pessoas com deficiência é de extrema eficácia para a promoção da qualidade de vida das mesmas, segundo Melo e López (2002) "é a oportunidade de testar seus limites e potencialidades, prevenir as enfermidades secundárias a sua deficiência e promover a integração social do indivíduo".

A reabilitação é um processo que diz respeito ao desenvolvimento humano e às capacidades adaptativas nas diferentes fases da vida. Abrange os aspectos funcionais, psíquicos, educacionais, sociais e profissionais (BRASIL, 2008).

Os objectivos da reabilitação é assegurar à pessoa com deficiência, independente da natureza ou da origem da deficiência, a mais ampla participação na vida social e ainda proporcionar a maior independência possível em atividades da vida diária.

Conforme Pereira (2009), quando abordamos o termo reabilitação de pessoas com deficiência, a intencionalidade tanto pode ser direcionada a restauração de funções quanto pode vincular-se ao processo de participação social da pessoa com deficiência.

Dessa forma, as ações de reabilitação visam ao desenvolvimento de capacidades, habilidades e recursos pessoais para promover a independência e a integração social das pessoas com deficiência, frente à diversidade de condições e necessidades.

Assim, através do desporto adaptado estamos proporcionando condições para que essa população também se reconheça como ser humano e busque seu desenvolvimento de forma lúdica e prazerosa. Grandes benefícios são evidenciados com a prática desportiva por pessoas com deficiência, entre estes podem ser destacados, a 
reabilitação física, psicológica e social, melhoria geral da aptidão física, grandes ganhos de independência e autoconfiança para a realização de atividades da vida diária, além de uma melhora do autoconceito e da auto-estima dos praticantes.

Com isso o objectivo deste estudo foi de realizar uma revisão de literatura sobre a reabilitação de pessoas com deficiência através do desporto adaptado.

\section{METODOLOGIA}

O presente estudo se caracteriza por ser uma revisão da literatura baseada em referências nacionais e internacionais obtidas através da base de dados da CAPES, Pubmed e Scielo, como também na base de dados da biblioteca da Faculdade de Desporto da Universidade do Porto - Portugal. A pesquisa foi feita com ano limite de 1990 a 2009. Foram utilizadas as palavras chaves: reabilitação, desporto adaptado, benefícios físicos, psicológicos e sociais do desporto adaptado.

\section{ORIGEM DO ESPORTE ADAPTADO}

Por volta de 1870, anteriormente ao movimento pós-guerra, já se tinha conhecimento sobre o desporto adaptado para pessoas surdas, iniciava nos Estados Unidos as primeiras participações desportivas organizadas por escolas especiais (WINNICK,2004; ARAÚJO, 1997). Em Paris, 1924, aconteceram os "Jogos do Silêncio", reunindo atletas de diversos países (WINNICK, 2004). Para Adams et al. ( 1985), a prática desportiva para pessoas com deficiência física na Alemanha teria se iniciado em 1918, para amenizar os horrores da Primeira Guerra Mundial e 1932 em Glasgow no Reino Unido Inglaterra existiu uma associação de jogadores de golfe, abarcando amputados unilaterais de membros superiores (COMITE OLÍMPICO ESPANHOL, 1994; MAUERBERG-DE-CASTRO, 2005).

O término da Segunda Guerra Mundial (1939-1945) foi um marco para a evolução do desporto adaptado para pessoas com deficiência, muitos soldados retornavam aos seus países, traziam em seu corpo marcas que jamais esqueceriam. O pós-guerra deixou muitos soldados mutilados, com distúrbios motores, visuais e auditivos, isso fez com que seus governos tomassem uma série de providências sobre a qualidade de vida desses indivíduos, com isso muitos começaram a ter acesso as práticas esportivas e atividades físicas adaptadas como forma de tentar minimizar as adversidades causadas pela guerra.

Em países da Europa e nos Estados Unidos essa preocupação foi maior, e os resultados dela foram evidenciados notavelmente. Aos poucos ex-veteranos de 
guerra começaram a obter grande êxito em atividades esportivas, as mais visadas foram: o basquete sobre cadeira de rodas e o atletismo. Para Mattos (1994), enquanto na Inglaterra o objetivo maior era a reabilitação pelo desporto, nos Estados Unidos a meta final era a competição.

Em Aylesbury na Inglaterra, o médico neurologista e neurocirurgião, alemão de origem judaica, Sir Ludwig Gutmann e seus colegas do Stoke Mandeville Hospital, obtém inúmeros sucessos com a reabilitação de ex-combatentes através de jogos desportivos com cadeira de rodas (MATTOS, 1990; VARELA, 1991; MAUERBERG-DE-CASTRO, 2005; GORGATTI; GORGATTI, 2005).

O primeiro programa em cadeira de rodas do hospital foi iniciado em 1945 com o objectivo de trabalhar o tronco e os membros superiores e diminuir o tédio da vida hospitalar (ARAÚJO, 1997). Sobre isso, o Jornal SuperAção (1988) divulga que: "Os primeiros resultados dessa prática relatam que, em um ano de trabalho, o Dr. Gutmann conseguiu preparar seis paraplégicos para o mercado de trabalho e reconheceu que as atividades desportivas, como medida terapêutica, eram importantes para a reabilitação psicossocial dos deficientes".

Em 1948 aconteceram os Jogos Olímpicos de Verão, em Londres, Inglaterra, e Sir Ludwig Gutmann, que passou a sonhar com uma olímpiada especial que reunisse milhares de deficientes em torno do desporto, aproveita o evento e cria paralelamente os primeiros jogos de Stoke Mandeville para paraplégicos, estes jogos contaram com a participação de 16 atletas ingleses nas modalidades de: arco e flecha, tiro ao alvo e arremesso de dardo (ARAÚJO, 1997; WINNICK, 2004; CIDADE; FREITAS, 2002; GORGATTI; GORGATTI, 2005).

Após isso em 1952, os jogos desenvolveram-se para a primeira competição internacional de desporto em cadeira de rodas para deficientes físicos, com cento e trinta participantes, equipes dos EUA, Inglaterra e Holanda (ARAÚJO, I997; WINNICK, 2004; CIDADE; FREITAS, 2002).

Os $9^{\circ}$ Jogos de Stoke Mandeville foram realizados em Roma na Itália em 1960, logo após o encerramento dos Jogos Olímpicos, este evento contou com cerca de duzentos e trinta atletas de vinte e três países, contou com o apoio do Comitê Olimpíco Italiano (COI), tendo como madrinha dos jogos a primeira dama italiana, Sra . Carla Grounchi. Este evento marca o envolvimento político e social do mundo todo com os jogos para pessoas com deficiência.

O nome "Paraolimpíadas" foi cunhado durante a Olimpíada de Tóquio, em 1964 e surgiu com uma paciente paraplégica do Stoke Mandeville Hospital, Alice Hunter, que escreveu seu relato intitulado "Alice of the Paralympiad" (Alice das Paraolímpiadas), para uma revista de desportos "The Cord Journal of the paraplegics", na época o termo foi associada a paraplegia e posteriormente foi cunhado 
para batizar os Jogos Paraolímpicos (PARALYMPIC SPIRIT apud MAUERBERG-DECASTRO 2005).

No Brasil, a história do desporto adaptado surge em 1958, quando foram fundados dois clubes de desporto em cadeira de rodas, um em São Paulo e outro no Rio de Janeiro. Foram fundados por Sérgio Serafim Del Grande e Robson Sampaio de Almeida, que trouxeram a ideia do desporto adaptado ao Brasil após retornarem de tratamentos de reabilitação em hospitais americanos, onde adquiriram o conhecimento da prática do desporto em cadeira de rodas (ARAÚJO, 1997; CIDADE; FREITAS, 2002; MAUERBERG-DE-CASTRO, 2005; GORGATTI; GORGATTI, 2005).

A participação brasileira nos Jogos Paraolímpicos só aconteceu em 1972, em Heidelberg, Alemanha. A modalidade foi a bocha, mas sem conquista de medalhas, em 1976, nos Jogos de Toronto, Canadá, nessa mesma modalidade, Robson de Almeida e Luis Carlos Coutinho conquistam as duas primeiras medalhas paraolímpicas (prata) para o Brasil (ARAÚJO, 1997; MAUERBERG-DE-CASTRO, 2005).

De 1984 a 2008 os Jogos Paraolímpicos sempre contaram com a participação do Brasil, neste período o número de medalhas conquistadas pelos atletas paraolímpicos ultrapassa ao dobro das conquistadas por atletas sem deficiência nos Jogos Olímpicos.

\section{BENEFÍCIOS FÍSICOS, PSICOLÓGICOS E SOCIAIS DO DESPORTO ADAPTADO}

A prática de actividades desportivas para pessoas com deficiências, além de proporcionar todos os benefícios para seu bem estar e qualidade de vida, também é a oportunidade de testar seus limites e potencialidades, prevenir as enfermidades secundárias à sua deficiência e promover a integração social e a reabilitação da pessoa com deficiência.

Souza (2004) apud Melo e López (2002) enfatiza que o desporto adaptado deve ser considerado como uma alternativa lúdica e prazerosa, sendo parte da reabilitação de pessoas com deficiências físicas. Através dessa prática desportiva lúdica e prazerosa para o indíviduo, estas atividades surgem como facilitadoras para a melhoria da qualidade de vida.

desporto adaptado é indicado desde a fase inicial do processo de reabilitação. Os indivíduos têm a oportunidade de vivenciar sensações e movimentos, que muitas vezes não realizaram pela limitação física ou por barreiras sociais e ambientais. 
desporto para pessoas com algum tipo de deficiência, iniciou como uma tentativa de colaborar no processo terapêutico delas e logo ganhou muitos adeptos (GORGATTI; GORGATTI, 2005). Como exemplo disso, o deficiente físico que inicia no desporto adaptado busca principalmente a reabilitação (recomendação médica) e a oportunidade de engajamento social com pares sob as mesmas condições (WHELLER et al., 1999). Dessa forma o desporto adaptado é uma ferramenta importante na reabilitação de indivíduos com deficiência, pelos benefícios motores, psicológicos e sociais, além de terem como objetivos o lazer e a competição, que são considerados aceleradores do processo de reabilitação.

Seus benefícios são muitos, como a melhoria da autoconfiança para a realização das atividades diárias, valorização pessoal, autoestima, melhora da condição física, aprimoramento das capacidades físicas gerais e prevenção de deficiências secundárias e reabilitação motora (BRAZUNA e MAUERBERG-DE-CASTRO, 2002; GORLA et al., 2007; GORGATTI et al., 2008).

Nahas (2006, p. 139) afirma que: "As atividades físicas e desportivas regulares podem reduzir os sintomas de ansiedade e depressão, promover a socialização e aumentar os níveis e bem-estar geral das pessoas com deficiência".

Standal e Jespersen (2008), evidenciaram em seu estudo com vinte e um indivíduos com deficiência física de dois programas de reabilitação da Noruega, a interação entre os participantes e a elevação da possibilidade de reabilitação através das atividades desportivas por alunos com deficiência inseridos nestes programas.

Labrocini et al. (2000), em estudo realizado para avaliar a reabilitação através do desporto, com trinta pessoas com deficiência física iniciantes no desporto adaptado, sendo quinze iniciados do basquete e quinze inicados da natação, os autores relatam grandes benefícios afetivos, sociais (baixa incidência a depressão, alto vigor e melhora nos relacionamentos) e de lazer desses indíviduos. Segundo os autores as mudanças de comportamento evidenciadas demonstram a importância do desporto adaptado na vida desses indivíduos, que passaram a relacionar-se de maneira diferente com a sociedade onde estão inseridos, também demonstram mais facilidade para enfrentar as dificuldades ou barreiras encontradas em seu cotidiano. E consideram que a integração que o desporto adaptado proporcionou, foi importante para os próprios atletas e também para as pessoas as quais eles se relacionam diariamente. Este estudo mostra que o desporto pode trazer para a pessoa com deficiência física, uma melhor integração social e adaptação a sua condição física.

Giacobbi Jr. et al. (2008), analisando a qualidade de vida de vinte e seis indivíduos (doze masculino e quatorze feminino) com deficiência física participantes de um torneio de basquete em cadeira de rodas nos Estados Unidos, observaram ganhos em sua percepção psicológica, afetiva e social. Também obtiveram benefícios na saúde associados ao seu envolvimento à atividade física. 
Harada e Siperstein (2009), realizaram um estudo com quinhentos e setenta e nove atletas americanos com deficiência intelectual das Special Olympics e evidenciaram que as actividades desportivas são fundamentais para as interações sociais desses indíviduos e suas famílias. Além de proporcionar benefícios afetivos e sociais, as atividades proporcionam ganhos físicos e motores.

Tsutsumi et al. (2004), citam que as atividades motoras em meio líquido visam o desenvolvimento cognitivo, afetivo, emocional e social, sendo mencionadas como um excelente meio de execução motora, favorecendo o desenvolvimento global do indivíduo com deficiência física.

Sporner et al. (2009), em estudo com cento e trinta e dois indíviduos participantes do National Veterans Wheelchair Games (NWWG) e do $20^{\circ}$ Winter Sports Clinic (WSC) nos Estados Unidos, recomendam que a participação de pessoas com deficiência nesse tipo de eventos podem promover benefícios psicológicos e também proporcionar a reabilitação física em pessoas com deficiência.

Martin (2006), relata em seu estudo com cento e doze atletas com deficiência, que o desporto é um importante aspecto para a ampliação das relações sociais desses indíviduos. Através das relações sociais dentro do desporto é possível promover a qualidade de vida de pessoas com deficiência.

Em estudo realizado na Universidade Federal de Santa Maria por Borges et al. (2007), onde 23 alunos com deficiência física participaram da adaptação de regras e fundamentos do basquete e do futebol, os autores afirmam que através da prática desportiva para pessoas com deficiência, foi possível observar melhoras significativas em relação ao aspecto afetivo-social, na cooperação e o respeito entre os participantes durante as práticas desportivas e também em relação ao desenvolvimento motor dos participantes.

Soler (2005) aborda que os esportes servem para aumentar o sentimento de autonomia. Os jogos servem para explorar o mundo que os rodeia; reforça a convivência, o alto grau de liberdade faz com que os relacionamentos fiquem mais saudáveis.

Steinberg e Bittar (1993) consideram que o desporto deveria ser sempre, parte da reabilitação de um indivíduo, lembrando que todos indivíduos apresentam um grau de potencial residual que deve ser estimulado em busca de uma vida mais saudável e digna.

\section{CONSIDERAÇÕES FINAIS}

Desporto para pessoas com deficiência ganhou extrema relevância em nosso país e no mundo, novos adeptos, novas competições, novas modalidades, novas metodologias e um grande interesse científico, contribuiram para o engrandecimento do desporto adaptado para pessoas com deficiências.

Os benefícios da prática desportiva pela pessoa com deficiência são facilmente perceptíveis, melhorias em seu aspecto fisico-motor, psicológico e social são eviden- 
ciados por grande parte de professores e estudiosos da área do desporto adaptado e contribuem possitivamente para a qualidade de vida da pessoa com deficiência.

Contudo, apesar desses avanços e benefícios, percebe-se que ainda existem muitos espaços a serem conquistados. Dentre estes, destaca-se a formação profissional para atução com pessoas com deficiência, que ainda carece de incremento na qualidade; melhorias e concretizações em prol da Inclusão, e também em termos de oportunidades de prática desportiva, que percebe-se que indivíduos com deficiência ainda encontram muitas dificuldades e se deparam com falta de apoio, acessibilidade e preconceito para começar e se manter realizando uma modalidade desportiva adaptada.

Por fim, o esporte adaptado ainda tem muito a proporcionar para pessoas com deficiência. Ao que parece, o caminho da educação e da conscientização pode ser de grande importância para continuar a auxiliar na melhoria da qualidade de vida desta população.

\section{The rehabilitation of persons with disabilities through adapted sport.}

ABSTRACT: Sport is an important means of physical rehabilitation, psychological and social development of people with some type of disability. It is modified and adapted methodologies, rules, materials for the participation of people with disabilities. The purpose of this study was to review the literature on the rehabilitation of disabled people through sport adapted, based on national and international references obtained from the database of CAPES, Pubmed, SciELO and the database of the library of the Faculty of Sport at the University of Porto - Portugal. It was observed that the benefits of sport are evident for most of scholars in the field of adapted sport and contribute to the quality of life in this population.

KEYWORDS: Rehabilitation; adapted sport; sports practice; disabled Persons.

\section{La rehabilitación de las personas con discapacidad a través del deporte adaptado}

RESUMEN: El deporte es un medio importante de la rehabilitación física, psicológica y social a las personas con algún tipo de discapacidad. Se trata de modificar y adaptar metodologías, normas, materiales para la participación de las personas con discapacidad. El propósito de este estudio fue revisar la literatura sobre la rehabilitación de las personas con discapacidad a través del deporte adaptado, basado en referencias nacionales e internacionales obtenidos a partir de la base de datos de la CAPES, PubMed, Scielo y la base de datos de la biblioteca de la Facultad de Deporte en la Universidad de Porto - Portugal. Se observó que los beneficios del deporte son evidentes para la mayoría de los estudiosos en el ámbito del deporte adaptado y contribuir a la calidad de vida en esta población.

PALABRAS CLAVE: Rehabilitación; deporte adaptado; práctica deportiva; personas con discapacidad. 


\section{REFERÊNCIAS}

ADAMS. R. C. et al. Jogos, esportes e exercícios para deficiente físico. 3. ed. São Paulo: Manole, 1985.

ARAÚJO, P. F. Desporto Adaptado no Brasil: origem, institucionalização e atualidades. 1997. I40f. Tese (Doutorado) Faculdade de Educação Física, Universidade Estadual de Campinas, Campinas, 1997.

BRASIL. A pessoa com deficiência e o Sistema Único de Saúde. Ministério da Saúde. Secretaria de Atenção à Saúde. Departamento de Ações Programáticas Estratégicas. 2. ed. Brasília: Editora do Ministério da Saúde, 2008.

BORGES, F. P. et al. Futebol e basquete: adaptações de regras para o ensino-aprendizagem de alunos com necessidades educacionais especiais. Revista Digital, Buenos Aires, v. 12, n. I |2, set. 2007. Disponível em: <http://www.efdeportes.com/efd I I /ffutebol-ebasquete-para-alunos-com-necessidades-especiais.htm>. Acesso em: 13 Jul. 2009.

BRAZUNA, M. R.; CASTRO, E. M. A trajetória do atleta portador de deficiência física no esporte adaptado de rendimento: uma revisão da literatura. Revista Motriz, Rio Claro, v. 7, n.2, p. I|5-123,jul-dez, 2001.

CIDADE, R. E. A.; FREITAS, P. S. Introdução à Educação Física e ao Desporto para Pessoas Portadoras de Deficiência. Ied. Curitiba: Ed. UFPR, 2002.

COMITÊ OLÍMPICO ESPANHOL. Esportes para minusválido físico, psiquico y sensoriales. España, 1994. 405p.

DUARTE, E.; WERNER, T. Conhecendo um pouco mais sobre as deficiências. In: Curso de atividade física e desportiva para pessoas portadoras de deficiência: educação à distância. Rio de Janeiro: UGF, v. 3, 1995.

GIACOBBI JR. ESPORTE entre portadores de deficiência. Jornal SuperAção, Rio de Janeiro, v. I, n. I, março 1988.

GORGATTI, M. G.; GORGATTI, T. O esporte para pessoas com necessidades especiais. In GORGATTI, M. G.; COSTA, R. F. (Orgs.), Atividade física adaptada: qualidade de vida para pessoas com necessidades especiais. Barueri: Manole, 2005. p. 532-568.

GORGATTI, M. G. et al. Tendência competitiva no esporte adaptado. Arquivos Sanny de Pesquisa e Saúde, v. 18, n. I, p. 18-25, 2008.

GORLA, J. l. et al. composição corporal em indivíduos com lesão medular praticantes de basquetebol em cadeira de rodas, Arquivos de Ciência da Saúde Unipar, Umuarama, v. I I, n. I, p. 39-44, jan./abr. 2007. 
HARADA, C. M.; SIPERSTEIN, G. N. The Sport Experience of Athletes With Intellectual Disabilities: A National Survey of Special Olympics Athletes and Their Families. Adapted Physical Activity Quarterly, v. 26, n. I, p. 68-85, 2009.

LABRONICI, R. H. D. D. O esporte como fator de integração do deficiente físico na sociedade. 1997. 98f. Dissertação (Mestrado em Neurociências). Escola Paulista de Medicina, Universidade Federal de São Paulo, São Paulo, 1997.

LABRONICI, R. H. D. D. et al. Esporte como fator de integração do deficiente físico na sociedade. Arquivos Neuro-Psiquiatria. v.58, n.4, p. 1092-1099, 2000.

LEVANDOSKI, G.; CARDOSO, A. S. Atletas de basquetebol em cadeiras de rodas da cidade de Florianópolis: uma análise descritiva das lesões dos praticantes. In: 6 Fórum Internacional de Esportes. Anais em CD, Florianópolis, jun. de 2007.

MARTIN, J. M. Psychosocial Aspects of Youth Disability Sport. Adapted Physical Activity Quarterly, v.23, n. I, p. 65-77, 2006.

MATTOS, E. Pessoas portadoras de deficiência física (motora) e as atividades físicas, esportivas, recreativas e de lazer. In: PEDRINELLI, V. J. (Org.) Educação Física e desporto para pessoas portadoras de deficiência. Brasília: MEC-SEDES, SESI, 1994. p. 75-85.

MATTOS, E. Esportes adaptados para portadores de deficiências físicas: implicações e aplicações. Anais III Simpósio Paulista de Educação Física Adaptada. São Paulo, 1990.

MAUERBERG-DE-CASTRO, E. Atividade Física Adaptada. Ribeirão Preto: Tecmed, 2005.

MELO, A. C. R.; LÓPEZ, R. F. A. O Esporte Adaptado. Revista Digital, Buenos Aires, v.8, n.5I, jul. 2002. Disponível em: <http://www.efdeportes.com/efd5 I/esporte.htm>. Acesso em: 20. nov. 2009.

NAHAS, M. V. Atividade Física, Saúde e Qualidade de Vida: conceitos e sugestões para um estilo de vida ativo. 4. ed., Londrina: Midiograf, 2006.

PEREIRA, S. O. Reabilitação de Pessoas com deficiência no SUS: Elementos para um debate sobre integralidade. 2009. I I 3f. Dissertação (Mestrado) Instituto de Saúde Coletiva, Universidade Federal da Bahia, 2009.

STANDAL , F.; JESPERSEN, E. Peers as Resources for Learning: A Situated Learning Approach to Adapted Physical Activity in Rehabilitation. Adapted Physical Activity Quarterly, v.25, n.3, p. 208-227, 2008.

STEINBERG, L. L.; BITTAR, A. Esportes e o portador de deficiência. In: DEPARTAMENTO DE ORTOPEDIA E TRAUMATOLOGIA. MANUAL DE MEDICINA FÍSICA E REABILITAÇÃO. São Paulo, 1993. p. 181 - 185.

SPORNER, M. L. et al. Psychosocial impact of participation in the National Veterans Wheelchair 
Games and Winter Sports Clinic. Disability \& Rehabilitation, v.3 I, n.5, p. 4I 0-4 I 8, 2009. TSUTSUMI, O. et al. Os Benefícios da Natação Adaptada em Indivíduos com Lesões Neurológicas. Revista Neurociências, São Paulo, v. 12, n. 2, abr.jun., 2004.

WHELLER, G. D. et al. Personal investment in disability sport careers: An international study. Adapted Physical Activity Quarterly, v. 16. n.3, p. 219-237, 1999.

WINNICK, J. P. Educação Física e Esportes Adaptados. 3. ed. Barueri: Manole, 2004.

Recebido: 19 mar. 2010

Aprovado: 10 nov. 2010

Endereço para correspondência

Vinícius Denardin Cardoso

Rua: Francisco Manoel 32, apto 201

Santa Maria-RS

CEP 970I5-260 